\title{
KI-gestütztes Recruiting - technische Grundlagen, wirtschaftliche Chancen und Risiken sowie ethische und soziale Herausforderungen
}

\author{
Gwendolin Wilke (D) Oliver Bendel
}

Eingegangen: 4. Oktober 2021 / Angenommen: 1. Februar 2022 / Online publiziert: 7. März 2022

(C) Der/die Autor(en) 2022

Zusammenfassung Bei der Rekrutierung von neuen Mitarbeitern und Mitarbeiterinnen setzen Firmen heute vermehrt auf die Unterstützung durch künstliche Intelligenz (KI). Die technischen Möglichkeiten reichen von Vorauswahlgesprächen mit Chatbots über die automatisierte Auswertung schriftlicher Bewerbungsdossiers hinsichtlich Fachkompetenz und Erfahrung bis hin zur maschinellen Analyse von Videobewerbungen zur Bewertung von Softskills wie Selbstvertrauen, Enthusiasmus oder Kommunikationsfähigkeit. Auch Lernplattformen werden zur Vorauswahl und Überprüfung eingesetzt. Der Artikel erklärt die technischen Grundlagen und Möglichkeiten des KI-gestützten Recruitings und zeigt wirtschaftliche Chancen und Risiken auf. Ethische und soziale Herausforderungen werden diskutiert und verständliche und umsetzbare Handlungsempfehlungen für Unternehmen unterbreitet, damit sie rechtliche Vorgaben und ethische Richtlinien einhalten und auf Kritik reagieren können.

Schlüsselwörter Künstliche Intelligenz $\cdot$ Recruiting $\cdot$ Robot Recruiting $\cdot$ People Analytics · Ethik

\footnotetext{
Gwendolin Wilke $(\bowtie)$

Hochschule für Wirtschaft, Institut für Wirtschaftsinformatik, Fachhochschule Nordwestschweiz, Riggenbachstrasse 16, 4600 Olten, Schweiz

E-Mail: gwendolin.wilke@fhnw.ch

Oliver Bendel

Hochschule für Wirtschaft, Institut für Wirtschaftsinformatik, Fachhochschule Nordwestschweiz, Bahnhofstrasse 6, 5210 Windisch, Schweiz

E-Mail: oliver.bendel@fhnw.ch
} 


\title{
AI-supported recruiting - technical background, economic opportunities and risks, ethical and social challenges
}

\begin{abstract}
When recruiting new employees, companies today increasingly rely on the support of artificial intelligence (AI). The technical possibilities range from preselection interviews with chatbots to the automated evaluation of written application dossiers in terms of professional competence and experience to the machine analysis of video applications to assess soft skills such as self-confidence, enthusiasm, or communication skills. Learning platforms are also used for pre-selection and review. The article explains the technical foundations and possibilities of AI-assisted recruiting and highlights economic opportunities and risks. It discusses ethical and social challenges and provides understandable and actionable recommendations for companies to comply with legal requirements and ethical guidelines and respond to criticism.
\end{abstract}

Keywords Artificial intelligence $\cdot$ Recruiting $\cdot$ Robot recruiting $\cdot$ People analytics $\cdot$ Ethics

\section{Einleitung}

Bei der Rekrutierung (dem Recruiting) von neuen Mitarbeitern und Mitarbeiterinnen setzen Firmen heute vermehrt auf die Unterstützung durch künstliche Intelligenz (KI). Wichtige Stichwörter in diesem Zusammenhang sind „Robot Recruiting“, „People Analytics“ und „HR Analytics“. Die technischen Möglichkeiten spannen sich von Vorauswahlgesprächen mit Chatbots über die automatisierte Auswertung schriftlicher Bewerbungsdossiers hinsichtlich Fachkompetenz und Erfahrung bis hin zu komplexen E-Assessments und zur maschinellen Analyse von Videobewerbungen zwecks Bewertung von Softskills wie Selbstvertrauen, Enthusiasmus oder Kommunikationsfähigkeit (Verhoeven 2020). ${ }^{1}$ Auch die Vorhersage des eigenen Mitarbeiterbedarfs kann intendiert werden.

Datenkonzerne wie Microsoft oder Facebook können schon heute Einträge in sozialen Netzwerken analysieren, um Aufschluss über Arbeitseinstellung, Teamgeist, Flexibilität oder Lernbereitschaft des Kandidaten oder der Kandidatin zu gewinnen. Online-Lernplattformen quantifizieren die Leistungsentwicklung von Benutzern - ggf. über mehrere Jahre hinweg - und machen so individuelle Potenziale oder psychologische Hürden sichtbar. Mit zunehmender Digitalisierung unserer Gesellschaft wird der Bürger oder Einwohner so zum lebenslangen Bewerber, dessen Daten hinsichtlich wirtschaftlicher Brauchbarkeit seiner Person permanent - mit Gewinn für alle Seiten - analysiert und evaluiert werden können.

\footnotetext{
1 Verhoeven legt den Schwerpunkt auf die vier Phasen Anziehung (durch KI unterstützte Auswahl und Steuerung von Online-Kanälen und intelligente Direct-Sourcing-Lösungen), Information (selbstlernende Chatbots), Bewerbung (Matching-Lösungen und Bewerbungsassistenten) und Auswahl (Analysetools für $\mathrm{CV}$ und KI-basierte Tests und Assessments).
} 
Diesen Chancen stehen allerdings erhebliche ethische, soziale und rechtliche $\mathrm{Ri}$ siken sowie demokratiepolitische Herausforderungen gegenüber. Diese reichen von der Gefährdung der informationellen Autonomie bzw. - rechtlich gesprochen - der informationellen Selbstbestimmung und der sicheren Aufbewahrung personenbezogener Daten über die Entstehung von Vorurteilen und Verzerrungen durch falsch konzipierte KI-Systeme bis hin zur Aufweichung demokratischer Grundwerte wie der Selbstbestimmung des Individuums durch unbewusste Verhaltensbeeinflussung.

Der vorliegende Artikel erklärt die technischen Grundlagen und Möglichkeiten des KI-gestützten Recruitings und zeigt wirtschaftliche Chancen und Risiken für die Anwender auf. Ethische und soziale Herausforderungen werden diskutiert und verständliche und umsetzbare Handlungsempfehlungen für Unternehmen unterbreitet, damit sie rechtliche Vorgaben und ethische Richtlinien einhalten sowie auf Kritik reagieren und Akzeptanz herstellen können.

\section{Technologien und Ansätze im KI-gestützten Recruitung}

Seit den 2000er-Jahren haben sich Wettbewerbsvorteile zunehmend von materiellen zu immateriellen Vermögenswerten verlagert. Damit ist Humankapital in vielen Unternehmen zu einem zentralen Gut geworden. KI-fähige Recruitingsysteme entwickeln sich im Zuge dessen von einem Nice-to-have zu einem Must-have (Black und van Esch 2020). Dennoch verwendeten bis 2018 nur ca. $31 \%$ der Unternehmen weltweit solche Tools (Deloitte 2018). Das vorliegende Kapitel beschreibt Technologien und Ansätze des KI-gestützten Recruitings, um einen Einblick in die Funktionsweise zu geben und so die Einsatzmöglichkeiten transparenter zu machen.

\subsection{Vom analogen zum KI-gestützten Recruiting}

Black und van Esch (2020) unterscheiden drei historische Entwicklungsstadien vom analogen bis hin zum heute verbreiteten KI-gestützten digitalen Recruiting. Traditionelles, analoges Recruting war bis Ende der 1990er-Jahre vorherrschend. Firmen publizierten Stellenausschreibungen vornehmlich in Zeitungen, wodurch die Reichweite stark beschränkt war. Da Anzeigen teuer waren, wurden Stellenausschreibungen kurz gehalten, sodass Anforderungsprofil und Angebot oft ungenau gerieten.

- Die sogenannte Reichweiten- und Reichhaltigkeitsgrenze (engl. „reach and richness frontier") konnte in einem ersten Stadium der Digitalisierung durch die Einführung von digitalen Job-Boards Anfang der 2000er-Jahre durchbrochen werden, denn Anzeigen in digitalen Medien sind wesentlich billiger und haben eine größere Reichweite als solche im analogen Bereich.

- In einem zweiten Stadium ab ca. 2010 konnten Anzeigen aus digitalen Job-Boards mittels Suchmaschinen automatisch aggregiert werden. Hinzu kam die Verbreitung von professionellen und sozialen Netzwerken wie LinkedIn und Facebook, was ein gezielteres Advertising für ausgesuchte Communities ermöglichte. Durch den Netzwerkeffekt explodierte die Anzahl der Bewerbungen pro Stellenausschreibung, während die der Bewerbungen unqualifizierter Personen zunahm. 
Dadurch wiederum stieg der Aufwand für Firmen, diejenigen Bewerber herauszufiltern, welche den geforderten Kriterien entsprachen.

- In einem dritten Stadium musste daher manuelles zunehmend durch automatisiertes Screening ersetzt bzw. ergänzt werden. Dies geschah anfangs auf Basis einer automatisierten Stichwortsuche in den Bewerbungsschreiben und wurde ab 2015 zunehmend durch KI-gestützte Methoden ergänzt. Diese haben in diesem Kontext den Vorteil, dass Eigenschaften von Kandidaten identifiziert werden können, welche nicht explizit ausgedrückt sind, wie Arbeitseinstellung oder Motivation.

KI-Methoden werden heute in der Ansprache (Outreach) von potenziellen Kandidatinnen und Kandidaten, in der Vorauswahl (Screening) von eingegangenen Bewerbungen und in der Bewertung (Assessment) der vorausgewählten Bewerbungen eingesetzt. Darüber hinaus kann der Prozess der Abstimmung und Kommunikation (Coordination) mit Bewerbenden verbessert werden.

\subsection{Künstliche Intelligenz im Recruitingprozess}

Warum sind KI-Methoden im Recruitingprozess nun so mächtig? Nach Poole und Mackworth (2010, S. 3) besteht ein KI-System aus intelligenten Computeragenten (Softwareagenten), die die Fähigkeit haben, ihre Umwelt wahrzunehmen, diese Wahrnehmungen mit Hilfe einer Wissensbasis und eines Datenanalysemoduls zu analysieren und dann mit einer „Handlung“ auf sie zu reagieren. Ziel ist es dabei, eine bestimmte vorgegebene Aufgabe mit den zur Verfügung stehenden Mitteln in einem iterativen Zyklus möglichst optimal zu erfüllen (s. Abb. 1a). Die Umwelt des Computeragenten kann dabei entweder eine Konzeptualisierung (eines Teils) der realen Welt sein oder eine rein virtuelle Welt.

Die virtuelle Umwelt des KI-Computeragenten im Recruitingprozess besteht z. B. aus den Benutzerprofilen von LinkedIn. Sein vorgegebenes Ziel könnte es sein, zur automatischen Unterstützung des Outreach-Prozesses die am besten passenden LinkedIn-Profile zu einem gegebenen Stellenprofil zu identifizieren. Um das Ziel zu erreichen, liest er alle Benutzerprofile ein, d.h. er „nimmt seine Umwelt wahr“.

a

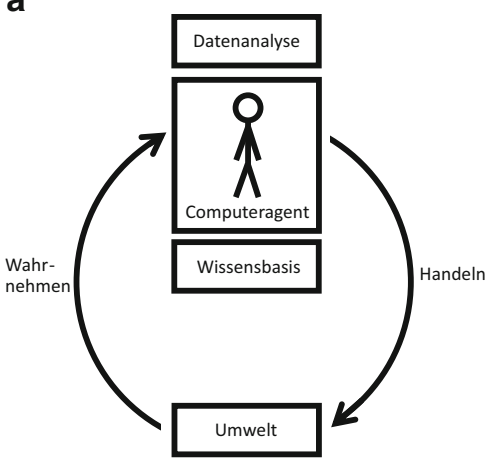

b

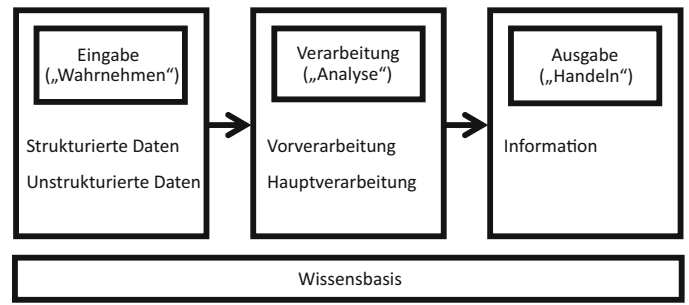

Abb. 1 a Computeragent, b Bausteine eines KI-Systems. (Adaptiert nach Paschen et al. 2020) 
Mit seinem Datenanalysemodul untersucht er sie, vergleicht sie mit dem in seiner Wissensbasis abgelegten Stellenprofil und sortiert sie hinsichtlich ihrer Eignung. Die besten Ergebnisse übergibt er an den Screening-Prozess (virtuelle Handlung), was den Zustand seiner virtuellen Umwelt beeinflussen kann. Denn sollte eine von ihm vorgeschlagene Person tatsächlich angestellt werden, ändert diese ihr Profil auf LinkedIn.

Intelligente Agenten besitzen also ein relativ hohes Maß an Autonomie. Viele von ihnen haben die Fähigkeit, mit ihren Handlungen auf die reale Welt Einfluss zu nehmen, etwa indem eine Person auf Grund der Analyse ihres LinkedIn-Profils tatsächlich angestellt wird. Die Hauptkomponente des KI-Systems, die Datenanalyse, erlaubt intelligentes und flexibles Lösen komplexer Probleme in kurzer Zeit. Sie hat durch die starke Zunahme an Rechenkapazität und den dadurch ermöglichten stärkeren Einsatz maschinellen Lernens seit Beginn der 2000er-Jahre einen enormen Aufschwung erfahren. Das Zusammenspiel von schnellem, flexiblem Problemlösen und einem hohen Grad an Autonomie ermöglicht eine Unterstützung des Recruitingprozesses in einem vorher nicht dagewesenen Ausmaß.

\subsection{Die Bausteine eines KI-Systems}

Nach Paschen et al. (2020) können die Eingabedaten („Wahrnehmen“) sogenannte strukturierte Daten sein, wie Einträge in der Datenbank einer Recruitingsoftware, oder unstrukturierte Daten, wie Profilbeschreibungen auf LinkedIn oder aufgezeichnete Videointerviews (s. Abb. 1b). Die Verarbeitung („Analyse“) der Eingabedaten gliedert sich in Vorverarbeitung und Hauptverarbeitung.

- In der Vorverarbeitung findet man häufig Methoden des Natural Language Processing (NLP), welche es erlauben, komplexe Bedeutungen aus Text zu extrahieren, etwa zur Arbeitseinstellung eines Bewerbers. Methoden der Computer Vision wiederum ermöglichen es, Bilder zu erkennen und auszuwerten. So kann die Mimik einer Bewerberin in einem Videointerview hinsichtlich Emotionen und Stimmungen wie Stress, Selbstsicherheit oder Begeisterung analysiert werden.

- In der Hauptverarbeitung werden mehrheitlich Methoden des maschinellen Lernens (engl. ,machine learning“) eingesetzt, wie Deep Learning oder Support Vector Machines. Aber auch Methoden des automatisierten Schließens (engl. ,automated reasoning“) und Problemlösens (engl. ,problem solving“) finden Anwendung, um Muster zu erkennen oder Bewerber auf Basis ihrer Eigenschaften zu klassifizieren. Beispielsweise können Informationen aus Bewerbungstexten und Videointerviews genutzt werden, um Persönlichkeitsprofile zu erstellen. ${ }^{2}$ Wenn Machine-Learning-Modelle genutzt werden, müssen diese jedoch vorgängig ,trainiert" werden. Um etwa den Persönlichkeitsfaktor „Extraversion“ in einem Videointerview automatisch identifizieren zu können, müssen dem Modell vorgängig Beispiele extrovertierter Persönlichkeiten ,gezeigt“ werden. Viele Softwareanbieter bieten hier vortrainierte Modelle an, welche direkt anwendbar sind. Dies ist

\footnotetext{
${ }^{2}$ Ein hierfür oft genutztes Modell ist das Fünf-Faktoren-Modell der Persönlichkeitspsychologie (Hickman et al. 2021; Norman 1963).
} 
immer dann möglich, wenn die gesuchten Muster allgemeingültig und nicht firmen- oder positionsspezifisch sind.

Um eine Analyse durchführen zu können, ist Hintergrundwissen, d.h. eine Wissensbasis, erforderlich. Soll bspw. ein erstelltes Persönlichkeitsprofil genutzt werden, um potenzielle Kandidaten im Assessmentschritt automatisiert zu bewerten, muss bekannt sein, welche der identifizierten Persönlichkeitsfaktoren in welchen Ausprägungen für die ausgeschriebene Position wünschenswert sind, und sie müssen in der Wissensbasis zugreifbar sein; soll etwa ein Chatbot Auskünfte über Anstellungsbedingungen und Unternehmensstruktur liefern, müssen die entsprechenden Informationen ebenfalls in der Wissensbasis abgelegt sein. Diese beinhaltet also Daten, die das „Handeln“ des Agenten (hier das Bewerten von Kandidaten oder die Beantwortung der Frage) beeinflussen. Sie kann dabei strukturierte oder unstrukturierte Daten umfassen, sie kann generisch sein oder aufgabenspezifisch, und sie kann manuell oder (teil-)automatisch befüllt werden.

Im Ausgabeschritt (,Handeln“) werden die in der Analyse gefundenen Erkenntnisse so aufbereitet, dass eine Interaktion des Agenten mit der Umwelt möglich wird. So muss ein Chatbot eine Antwort auf eine gestellte Frage in natürlicher Sprache formulieren können, um vom menschlichen Gegenüber verstanden zu werden. Dies kann mit Methoden der Natural Language Generation (NLG) erfolgen. Ferner können Bilder automatisch generiert werden (engl. ,image generation“), um gefundene Resultate verständlich zu kommunizieren.

\subsection{KI-basierte Textanalyse}

Eine der wichtigsten Methoden des KI-gestützten Recruitings ist die KI-basierte Textanalyse. Diese geht über das simple Durchsuchen des Textes nach passenden Schlüsselwörtern und dessen Synonymen weit hinaus. Methoden des Natural Language Understanding (NLU) erlauben es bis zu einem gewissen Grad, Bedeutung aus textuellen Beschreibungen abzuleiten, d.h. den Text zu ,verstehen“ und über die offensichtlichen Aussagen hinaus zu ,interpretieren“. Hierfür bieten Softwarehersteller Programmierschnittstellen (APIs) oder fertige Services an. ${ }^{3}$

Mittels KI-basierter Textanalyse kann nicht nur der Inhalt eines Textes, sondern auch die Wortwahl oder der Satzbau analysiert werden, um Hinweise auf erfolgsrelevante Eigenschaften zu erhalten, sodass semantische und syntaktische Ebene betroffen sind. So ist eine exakte Formulierung komplexer Zusammenhänge bei Verwendung einschlägiger Sachbegriffe offenbar ein Indiz für langjährige Erfahrung auf einem Wissensgebiet.

Im Outreach-Prozess kann Textanalyse bei Kandidatenprofilen in Netzwerken wie LinkedIn genutzt werden. Im Screening-Prozess dient sie u. a. der Vorauswahl von relevanten Bewerbungsdossiers, im Assessment-Prozess der Erstellung von Persön-

\footnotetext{
3 Siehe etwa IBM Watson Natural Language Understanding (https://cloud.google.com/natural-language), Microsoft Azure LUIS (https://azure.microsoft.com/de-de/services/cognitive-services/language-under standing-intelligent-service), Amazon Comprehend (https://aws.amazon.com/de/comprehend) oder Google Natural Language API (https://cloud.google.com/natural-language).
} 
lichkeits- und Kompetenzprofilen. Im Coordination-Prozess kann sie die Kommunikation mit Kandidaten erleichtern, indem etwa E-Mails oder die Kommunikation mit Chatbots auf ihre Bedeutung hin analysiert werden. Insbesondere Chatbots sind auf ein gutes Verständnis von Text, Subtext, Kontext etc. angewiesen, um ein positives Erlebnis und einen sinnvollen Austausch zu ermöglichen.

\subsection{Chatbots und Voicebots}

Ein Chatbot bzw. Voicebot (Voice Assistant) ist ein Computeragent, welcher menschliche Kommunikation auf Basis von natürlichsprachlichem geschriebenem Text bzw. natürlichsprachlicher gesprochener Sprache nachahmt und somit intelligente und intuitive Mensch-Maschine-Kommunikation (engl. ,human-computer interaction“, HCI) ermöglicht (Bendel 2019). Er interagiert also auf intelligente Weise mit seiner Umwelt (dem Menschen) und ist damit ein typisches Beispiel eines intelligenten Agenten, also eines bestimmten KI-Systems (s. Abb. 1).

Chatbots sind Dialogsysteme, die im Kern Methoden des NLP nutzen: das NLU, um geschriebene Sätze zu ,verstehen“, eine Dialogsteuerung, um zu entscheiden, was als Nächstes im Kontext des aktuellen Dialogstatus zu tun ist, und die ResponseGeneration-Einheit, um den Inhalt der gefundenen Antwort mittels NLG in natürlicher Sprache zu vermitteln (Adamopoulou und Moussiades 2020). Während die meisten Chatbots bis anhin manuell eingepflegte Dialogregeln benutzen, nimmt die Verwendung von datengetriebenen Systemen stetig zu. Diese gleichen Benutzereingaben mit Beispieldaten aus großen Dialogkorpora auf Basis statistischer Methoden ab, um die am besten passende Antwort zu finden. Sie sind dadurch flexibler, müssen jedoch noch mit firmenspezifischen Informationen angereichert werden. Einige Chatbots lernen mittels Reinforcement Learning aus jeder Kommunikation, um Informationen zu sammeln und die Qualität der Antworten kontinuierlich zu verbessern.

Beispiele für Out-of-the-Box-Chatbotsysteme für Recruiting sind XOR (https:// www.xor.ai), Olivia von Paradox (https://www.paradox.ai), Humanly (https:// humanly.io) und Espressive (https://www.espressive.com). Bekannte Beispiele für Voicebots sind Google Assistant (https://assistant.google.com), Siri von Apple (https://www.apple.com/de/siri/) und Alexa von Amazon (https://developer.amazon. com/de-DE/alexa) (Bendel 2021). Sie besitzen zwei zusätzliche Systemkomponenten: Automatic Speech Recognition (ASR), also die Umwandlung von Spracheingabe in geschriebenen Text, und die Umwandlung der generierten Textantwort zurück in synthetische gesprochene Sprache (Text-to-Speech). ${ }^{4}$

Chatbots und Voicebots spielen eine zentrale Rolle im Screening- und Coordination-Prozess, d.h. in der Vorauswahl und in der Kommunikation mit und Koordination von Bewerbern. Diese können Fragen an den Chatbot oder Voicebot richten, die bspw. den Bewerbungsprozess, das Unternehmen oder die ausgeschriebene Stelle betreffen. Chat- oder Voicebots können umgekehrt Anwendung finden, um Fragen an den Bewerber zu richten, etwa um fehlende Informationen im Bewerbungsdossier

\footnotetext{
${ }^{4}$ Beispiele solcher Komponenten sind Amazon Lex (https://aws.amazon.com/de/lex/) oder IBM Watson Speech to Text (https://www.ibm.com/cloud/watson-speech-to-text).
} 
automatisiert zu ergänzen (Black und van Esch 2020). So können die Bewerbungserfahrungen der Kandidaten verbessert werden, da die Bots rund um die Uhr zur Verfügung stehen. Unklarheiten bzgl. des Bewerbungsprozesses und der Rahmenbedingungen der potenziellen Anstellung oder der aktuelle Status der Bewerbung können einfach und unkompliziert abgefragt werden.

\subsection{KI-basierte Videoanalyse}

Immer häufiger werden Videointerviews durchgeführt, ob synchron oder asynchron. Ein synchrones Videointerview läuft wie ein herkömmliches Interview ab. Die Interviewpartner sprechen in Echtzeit miteinander, wobei eine Software für Videokonferenzen wie Zoom oder Skype genutzt wird. Für die ausschreibende Firma bietet sich der Vorteil, dass die Interviews aufgezeichnet und mit KI-basierten Methoden analysiert werden können. Für den Kandidaten oder die Kandidatin eröffnet sich Ortsunabhängigkeit. Zeitaufwändige Reisen für ein Vorauswahlgespräch können so entfallen. In einem asynchronen Videointerview werden die Fragen innerhalb von Videosequenzen formuliert und dem Bewerber zugestellt. Dieser zeichnet seine Antworten wiederum als Videosequenzen auf. Hierbei ergibt sich für ihn neben der Ortsunabhängigkeit noch der Vorteil einer relativen Zeitunabhängigkeit. Die Firma kann eine große Zahl von Interviews effizient führen und automatisch analysieren lassen.

Bei der Videointerviewanalyse können nicht nur explizit besprochene Inhalte automatisiert untersucht werden, sondern auch Persönlichkeitsfaktoren wie Beharrlichkeit, Risikobereitschaft, Selbstsicherheit oder Gewissenhaftigkeit aus der Analyse des Antwortverhaltens eines Kandidaten oder einer Kandidatin automatisch abgeleitet und daraus Persönlichkeitsprofile gefertigt werden. Dazu werden neben dem eigentlichen Inhalt einer Antwort bspw. Wortwahl, Satzbau, Artikulation, Stimmlage, Sprechgeschwindigkeit, Gesichtsausdruck, Mimik und sogar Mikromimik analysiert. ${ }^{5}$

Für die Inhaltsanalyse wird das aufgezeichnete Videointerview automatisch in geschriebenen Text transkribiert. Dieser kann dann wie oben beschrieben auf relevante Eigenschaften des Kandidaten oder der Kandidatin hin ausgewertet werden. Für die Analyse von Artikulation, Stimmlage und Sprechgeschwindigkeit wird die Tonspur herangezogen. Dabei werden akustische Merkmale wie Frequenzspektrum genutzt und mit den Kandidateneigenschaften in Beziehung gesetzt. Für die Analyse von Gesichtsausdruck und (Mikro-)Mimik verwendet man Verfahren der Bilderkennung. Spezialisierte Methoden, oft unter dem Schlagwort Affective Computing zusammengefasst, ermöglichen es, Emotionen und Stimmungen eines Kandidaten oder einer Kandidatin zu extrahieren. Hierbei kommen vornehmlich Deep Learning, Optic Flow Analysis, Hidden Markov Models oder Active-Appearance-Modelle zum Einsatz.

Um all diese Modelle und Methoden gebrauchen zu können, ist es nötig, sie mit Beispieldaten zu trainieren (überwachtes Lernen). So sammelt man für die Emotionsanalyse Beispiele für Gesichter, welche Ärger, Angst, Trauer, Überraschung, Freude etc. ausdrücken und entsprechend gekennzeichnet sind. Der Algorithmus

\footnotetext{
${ }^{5}$ Man unterscheidet hier Sprach-, Stimm- und Gesichtserkennung.
} 
lernt dann anhand dieser Beispiele neue Gesichter zu interpretieren, also solche, die das System noch nie ,gesehen“ hat. Da für die Lernphase eine sehr große Menge von Beispieldaten benötigt werden, existieren sogenannte Emotionsdatenbanken, welche diese bereitstellen. Eine Softwarelösung für KI-basiertes Recruiting, die Videoemotionsanalyse anbietet, enthält bereits trainierte Modelle, welche ,out of the box“" angewendet werden können.

\section{Wirtschaftliche Chancen und Risiken}

Im vorliegenden Kapitel werden wirtschaftliche Chancen und Risiken herausgearbeitet. Es kann dabei nur auf wenige Aspekte fokussiert werden. Die Zusammenstellung ist auf der Grundlage eines Brainstormings (mit anschließender Priorisierung mithilfe der Expertenerfahrung der Autoren) und von Literaturanalyse entstanden. Sie erhebt keinen Anspruch auf Vollständigkeit.

\subsection{Anschaffungskosten und laufende Kosten}

Der Einsatz von KI-basierten Recruitingsystemen zieht Anschaffungs- bzw. Bereitstellungskosten nach sich. Man kauft entweder entweder eine Lösung ein oder bezieht einen Dienst über die Cloud (z.B. Software-as-a-Service). Es ergeben sich laufende Betriebs- und Wartungskosten. Dazu kommen Ausgaben für die Einarbeitung der HR-Mitarbeiter für die Software sowie - je nach eingekaufter Lösung und eingesetzten Modulen - Zusatzkosten zum initialen Anlegen und Pflegen einer firmenspezifischen Wissensbasis. Bspw. sind bei Chatbots im Coordination-Prozess relativ umfassende Informationen zu den ,hard facts“ der Anstellungsbedingungen, des Unternehmens selbst und der ausgeschriebenen Position sinnvoll, um möglichst viele Fragen im Vorfeld abdecken zu können. Hierbei sind standardisierte Bewerbungsprozesse und Anstellungsbedingungen vorteilhaft. Beim Einsatz von Textanalyse im Outreach- oder Screening-Prozess hingegen müssen v. a. positionsspezifische Informationen zugreifbar sein, welche in relativ kurzer Zeit von der HR-Abteilung erfasst werden können. Anschaffungskosten und laufende Kosten sind nicht per se ein Nachteil, sondern müssen einfach einkalkuliert werden - und relativieren sich, wenn sich Einsparpotenziale ergeben.

\subsection{Einsparpotenziale durch Automatisierung}

Die stärkere Automatisierung des Recruitingverfahrens birgt Einsparpotenziale bei den Personalkosten, insbesondere in der Vorauswahl (im Screening) von Dossiers durch automatisierte Textanalyse und durch die Verkürzung oder Ersetzung von Vorgesprächen durch Chatbots im Coordination-Prozess. Bei Chatbots muss jedoch der Aufwand, eine entsprechende Wissensbasis anzulegen, mit dem eingesparten Kommunikationsaufwand abgewogen werden. Darüber hinaus können im Outreachund Screening-Prozess ggf. Kosten für professionelle Headhunter eingespart werden. Spesen für Bewerbungsfahrten fallen zumindest am Anfang weg, was je nach Fall den Behörden, den Unternehmen oder den Bewerbern hilft. 
Die Zeitspanne von Ansprache bis Einstellung (Time-to-Hire) kann durch KIgestützte Recruitingverfahren drastisch reduziert werden. Dies erhöht die Effizienz des Einstellungsprozesses gegenüber manuellem oder digitalem Recruiting ohne KI-Unterstützung und senkt massiv Kosten, da offene Stellen schneller und agiler besetzt werden können. Während in Zeiten des analogen Recruitings meist nur wenige Bewerbungen auf eine ausgeschriebene Position entgegengenommen und bearbeitet werden mussten, generiert der Netzwerkeffekt im digitalen Recruiting oft eine wahre Flut, was zunächst ein Nachteil zu sein scheint. So erhielt Johnson \& Johnson im Jahr 2017 rund eine Million Bewerbungen auf 28.000 Stellen (McIlvaine 2018). Insbesondere in Konzernen ist daher enormes Einsparungspotenzial durch KISysteme zu erwarten.

Noch wichtiger ist eine Senkung der Time-to-Hire in Branchen mit hoher Personalfluktuation. Hier ergibt sich neben Effizienzgewinn und Kostensenkung zusätzlich ein strategischer Vorteil im Wettbewerb um Humankapital. Eine Hotelkette beispielsweise befindet sich in vielen Fällen in einem permanenten Recruitingprozess. Eine qualifizierte Bewerberin, welche statt vier Wochen nur sieben Tage auf eine Zusage für eine vergleichbare Stelle warten muss, wird sich mit hoher Wahrscheinlichkeit für diejenige entscheiden, bei der eine schnellere Zusage eintrifft (Black und van Esch 2020).

\subsection{Innovationsförderung und Pionierstatus}

Das automatische Screening von Netzwerkprofilen ermöglicht ein aktives Sourcing, d.h. das direkte Ansprechen und etwaige Abwerben von Experten, welche zum gegebenen Zeitpunkt nicht nach einer Anstellung suchen (Guinan et al. 2014). Dabei werden Profile in LinkedIn, XING, Ryze, Facebook oder Twitter automatisch nach bestimmten Kriterien intelligent durchsucht oder mittels automatisierter Textanalyse aufgeschlossen (Campbell et al. 2020). ${ }^{6}$ Einige Firmen gehen heute bereits so weit, auf Bewerbungsdossiers komplett zu verzichten und stattdessen die Netzwerkprofile der Kandidaten zu screenen. Aktives Sourcing hat mit der zentralen Rolle von Humankapital für den Wettbewerbsvorteil in vielen Branchen massiv an Bedeutung gewonnen.

Der in einer Firma vorhandene Pool aus ehemals abgelehnten Bewerbungen wird mit steigendem Wettbewerbsdruck ebenfalls mehr und mehr als wertvolle Ressource erkannt: Eine in der Vergangenheit abgelehnte Bewerberin für eine bestimmte Position in der Firma kann durchaus die perfekte Passung für eine andere ausgeschriebene Position sein. Über solche Personen liegen bereits detaillierte Informationen vor, bspw. in Form von Dossiers, Korrespondenzen oder aufgezeichneten Videointerviews, welche direkt unter den neuen Gesichtspunkten ausgewertet werden. Das manuelle oder schlüsselwortbasierte Screening dieses Pools gestaltete sich bisher schwierig, da Bewerbungen oft nicht zentral vorgehalten werden und daher schwer vergleichbar waren. Der jeweils betriebene Aufwand stand in keinem Verhältnis zum Ergebnis. Diese Hürde kann durch die Flexibilität KI-gestützter Methoden vielfach

\footnotetext{
${ }^{6}$ Beispiele für Anbieter in diesem Bereich sind PandoLogic (https://www.pandologic.com) und Talenya (https://www.talenya.com).
} 
überbrückt werden. So können NLP-Verfahren oftmals auf (fast) beliebige Textformate angewendet werden, nachdem die entsprechenden Dossiers automatisiert zusammengeführt wurden.

KI kann Unternehmen auch dabei helfen, passendere Kandidaten gezielter anzusprechen, bspw. durch die zielgruppenspezifische Auswahl des Publikationskanals abhängig vom gewünschten Kandidatenprofil. Dabei platziert das System Stellenangebote auf Banner, auf Pop-ups, in E-Mails oder in anderen Texten so, dass eine optimale Reaktion erzielt wird. Textio (https://textio.com/products/recruiting/) nutzt KI-Verfahren, um die Wortwahl von Stellenanzeigen automatisch an die Eigenschaften der gesuchten Bewerber und an demografische Gegebenheiten anzupassen (McIlvaine 2018; Sharma 2018).

Im traditionellen Recruiting werden gemäß Testtheorie vornehmlich die Kriterien Objektivität, Reliabilität und Validität zur Beurteilung der Güte des Prozesses herangezogen, während das Kriterium der Akzeptanz durch die Bewerbenden häufig vernachlässigt wird. Eine positive Bewerbungserfahrung ist im Wettkampf um qualifizierte Mitarbeiter heute jedoch ein wichtiges Asset, da sich das Image einer Firma schnell über Mundpropaganda in sozialen Medien verbreitet. Bewerbende als Kunden zu behandeln, ist eine erfolgversprechende Strategie (Sharma 2018). Hier zeigt der Einsatz von KI-gestütztem Recruiting einen wesentlichen Vorteil: Eine Umfrage aus dem Jahr 2017 hat ergeben, dass ein wesentlicher Grund für eine negative Bewerbungserfahrung unzureichende Information bzw. Kommunikation während des Bewerbungsprozesses ist (Talent Board 2017) - ein Problem, welches bspw. mit Hilfe von Chatbots gemildert werden kann. Bewerbungen können darüber hinaus vereinfacht werden, indem statt eines ausgefüllten Formulars nur ein LinkedIn- oder XING-Profil angefragt wird. Das Bewerbungsformular kann dann automatisch vom KI-System ausgefüllt, eine fehlende Information vom Chatbot eingeholt werden. Solche und ähnliche Maßnahmen mögen dabei helfen, die Zufriedenheit der Bewerber mit dem Bewerbungsprozess massiv zu erhöhen.

\subsection{Abhängigkeit von Anbietern}

Setzt ein Unternehmen KI-Systeme ein, die es nicht selbst entwickelt, macht es sich abhängig von anderen Unternehmen, was sich meist als deutlicher Nachteil erweist. Dies betrifft sowohl die Zurverfügungstellung des Systems als auch seiner Komponenten. Zudem macht man sich abhängig von erfolgenden oder nicht erfolgenden technischen Entwicklungen, nicht zuletzt mit Blick auf die Konkurrenz. Sind KI-Verfahren im Recruiting beispielsweise etabliert, kommt man nicht umhin, die neueste Technologie einzusetzen, schon aus Reputationsgründen. Den Herstellern selbst kann es wirtschaftlich helfen, dass die Bewerber ihre Software kennenlernen, zumindest wenn deren Name genannt wird und die Nutzung befriedigend ist. Es ist insgesamt eher die Ausnahme, dass Personen, die nicht oder noch nicht zu einem Unternehmen gehören, etwas über dessen technische Infrastruktur erfahren. 


\section{Ethische, soziale und rechtliche Herausforderungen}

Im vorliegenden Kapitel werden ethische, soziale und - am Rande - rechtliche Herausforderungen des KI-gestützten Recruitings herausgearbeitet. Grundlage sind die identifizierten und skizzierten Technologien und Ansätze sowie die wirtschaftlichen Chancen und Risiken. Es wird von ethischen Ideen wie Gerechtigkeit und speziell von informationsethischen Konzepten wie der informationellen Autonomie und des digitalen Grabens ausgegangen und geprüft, ob diese tangiert werden. Die Zusammenstellung ist wiederum auf der Grundlage eines Brainstormings (mit anschließender Priorisierung mithilfe der Expertenerfahrung der Autoren) und von Literaturanalyse entstanden. Sie erhebt auch hier keinen Anspruch auf Vollständigkeit.

\subsection{Ersetzung des persönlichen Gesprächs und physischen Aufenthalts}

Der Einsatz von Chatbots und Voicebots (wie auch von Videointerviews) kann das persönliche Gespräch zwischen Einrichtungen und Bewerbern sowie den physischen Aufenthalt vor Ort ergänzen oder ersetzen, was ethische und soziale Aspekte hat. Wenn er es ersetzt, geht damit eine wichtige Komponente im Bewerbungsgespräch verloren. Im persönlichen Gespräch lernt der Bewerber nämlich einen oder mehrere Mitarbeiter kennen, erfährt damit indirekt oder direkt etwas über Kommunikation und Kultur im Unternehmen. Man hat die Möglichkeit, das Gegenüber jenseits der vorgesehenen Verläufe und Inhalte etwas zu fragen. Es ergibt sich ein erster Eindruck und im besten Falle eine erste Bindung. Dies alles ist auch ein Vorteil für das Unternehmen - es sollte Bewerber als Kunden behandeln im „war of talent“.

Wenn das Gespräch zwischen Mensch und Mensch durch eines zwischen Mensch und Maschine substituiert wird, kann dies dazu führen, dass die Qualität schwindet und die Akzeptanz leidet. „So kann es passieren, dass komplexere Fragen nicht oder falsch beantworten [!] werden, was zur Unzufriedenheit des Benutzers führen kann. Möglicherweise verliert das Unternehmen hierdurch schnell sein Image als moderner Arbeitgeber. Unklar ist auch, ob sich Bewerber diesen Systemen letztlich anvertrauen oder ob sie den Einsatz skeptisch betrachten und ihn sogar ablehnen“ (Mülder 2021). In diesem Sinne ist der Chatbot keine vollwertige und u. U. sogar unbefriedigende Lösung. Auch bei Videointerviews kann die Akzeptanz in Frage gestellt sein, insbesondere bei asynchronen, wo keine unmittelbare Interaktion und Kommunikation zwischen Menschen stattfindet.

Das Gespräch per Chatbot verläuft meist in einem eng gesteckten Rahmen - Small Talk wird zwar beherrscht, ist aber nicht im Vordergrund (wobei er dazu dienen kann, die Soft Skills abzufragen). Dies ist freilich im klassischen Bewerbungsgespräch nicht anders. Es tut sich die Chance auf, dass Persönliches, das nichts mit der Stelle zu tun hat, ausgeklammert wird. Man wird kaum einen Chatbot programmieren, der nach den Beziehungsverhältnissen und der Familienplanung fragt, und wenn doch, ist die Gefahr für das Unternehmen groß, dass dies an die Öffentlichkeit gelangt. Zudem kann der Dialog nicht nur vom Unternehmen, sondern auch vom Bewerber mitgeschnitten werden, was diesem eine gewisse Sicherheit bietet. 


\subsection{Verletzung der informationellen Autonomie und der Privatsphäre}

Mit dem Einsatz von Chatbots und der KI-basierten Analyse von Text und Gesicht (ggf. verbunden mit Emotionserkennung) bzw. Verhalten sowie der Nutzung von professionellen bzw. sozialen Netzwerken und Lernplattformen entstehen personenbezogene Daten, die im Unternehmen gespeichert werden. Dies ist bei klassischen Bewerbungsverfahren ebenso der Fall. Allerdings werden dort i.d.R. keine Gesichtsaufnahmen angefertigt (höchstens freiwillig in der Bewerbung geliefert) und in maschineller Weise verwertet, und es findet normalerweise keine automatisierte Stimmanalyse (etwa durch versteckte Systeme) statt. Qualität und Quantität sind also bei KI-gestützten Verfahren anderer Art. Grundsätzlich kann - ein zentraler informationsethischer Begriff - die informationelle Autonomie verletzt sein (im Recht spricht man von informationeller Selbstbestimmung). Insbesondere ist es dem Bewerber oder der Bewerberin kaum möglich, die ordnungsgemäße Verwendung der Daten zu kontrollieren und zu intervenieren.

Bei Videobewerbungen - ob KI-gestützt oder nicht - wird oft in den privaten Bereich des Bewerbers oder der Bewerberin eingedrungen. Nicht bei jedem Tool ist es ihm oder ihr möglich, auf einen virtuellen Hintergrund zu wechseln oder den Hintergrund unscharf zu stellen. Bei klassischen Bewerbungsgesprächen kommt man zum Unternehmen, lernt den Standort und das Gebäude kennen. In diesem Fall erfährt das Unternehmen eventuell etwas über die Wohnung und das Umfeld des Interessenten, ob das gewollt ist oder nicht. Theoretisch ist es möglich, mit Hilfe von Objekterkennung die entsprechenden Gegenstände zu identifizieren, zu klassifizieren und zu quantifizieren. Damit könnte man Einschätzungen zur Bildung, zum Wohlstand und zum Geschmack des Kandidaten abgeben, womit in seine Privatsphäre eingedrungen würde.

Es ist fraglich, ob es in moralischer Hinsicht zu vertreten ist, ein Gesicht einfach aufzunehmen und mit Hilfe von Technologien zu analysieren (Bendel 2018a). Die personenbezogenen Daten, so könnte man argumentieren, gehören der Person und dürfen nur unter speziellen und kontrollierten Bedingungen erhoben und verarbeitet werden. Kosinski und Wang (2017) haben auf den Eingriff in die Privatsphäre durch diese Form von Software aufmerksam gemacht. ${ }^{7}$ Bei der Analyse von Texten dürfte dieses Argument nicht gelten. Zumindest in unserem Kulturkreis gilt das Schriftliche nach seiner Weitergabe als etwas, was sich vom Urheber ablöst und beliebig untersucht werden darf. Es existiert das Urheberrecht, das die Verbreitung regelt, aber wohl kein moralisches Recht des Urhebers, vor Textanalyse geschützt zu sein. Allerdings könnte man argumentieren, dass hier persönliche und vertrauliche Dokumente vorliegen sowie Vertrauen missbraucht und Unwissen ausgenutzt wird.

Der Einsatz von Machine Learning inkl. Deep Learning wirft weitere Fragen auf. Der Bewerber wird zum Trainingspartner des Systems, ob er das möchte oder nicht. Zudem fließen seine Daten und Informationen ein und werden bei weiteren Gesprächen verwendet. Zwar wird man kaum auf vorherige Gesprächspartner schließen können, und auch das Urheberrecht dürfte kaum greifen - dennoch kann man das

\footnotetext{
${ }^{7}$ In rechtlicher Hinsicht ist noch - bezogen vor allem auf die Speicherung und Verbreitung - auf das Recht am eigenen Bild hinzuweisen.
} 
Auswerten der Dialoginhalte und des Kommunikationsverhaltens beanstanden. Zudem kann sich das System je nach vorangegangener Bewerberschaft in eine für den Bewerber positive oder negative Richtung entwickeln. Es ,,besteht die Gefahr, dass allein durch Erfahrungslernen aus vergangenen Daten falsche Schlussfolgerungen gezogen werden“ (Mülder 2021).

\section{3 (Ohn-)Mächtigkeit der Systeme}

Ein heikler Punkt ist, dass Soft- und Hardware andere und noch mehr Eigenschaften zu finden scheinen als Menschen und - dies berührt soziale und ethische Aspekte eine gewisse Mächtigkeit besitzen. Sie scheinen herauszulesen, was wir überlesen, zu sehen, was wir übersehen, zu hören, was wir überhören. Dies lässt sich etwa bei der Bestimmung von Alter und Geschlecht feststellen. Wir lassen uns von Kleidung und Frisur sowie Habitus beeinflussen, während das Gesichtserkennungssystem sich eben auf das Gesicht konzentriert (Bendel 2018a). Wenn das System einer Frau ins Gesicht sagt, dass sie ein Mann ist (oder umgekehrt), wird sie (oder ihn) das nicht immer erfreuen. Bei der Bewerbung könnte dies durchaus eine Rolle spielen, etwa wenn das Unternehmen weibliche oder männliche Erscheinungsformen wünscht in Gastronomie und Hotellerie sowie im Unterhaltungsgewerbe nicht unüblich. Im klassischen Bewerbungsgespräch wird diese Auswahl ebenso vorgenommen, doch womöglich weniger streng.

Zugleich sind manche KI-Systeme blind gegenüber einer Vielzahl von menschlichen Ausdrücken und gegenüber lokalen Eigenheiten und Gepflogenheiten. Darin zeigt sich sozusagen ihre Ohnmächtigkeit. Sie können mit Hilfe von Mimikerkennung und Textanalyse (etwa eines Chatverlaufs) gewisse Emotionen erkennen, gewisse jedoch nicht, und man muss berücksichtigen, dass sich der Benutzer einem System gegenüber bewusst oder unbewusst anders verhält als einem Menschen, etwa indem er die Mimik reduziert. Dies lässt sich ein Stück weit beheben, indem man das Interview mit Hilfe von Aufzeichnungen der Interviewer führt (oben wurde von synchronen Videointerviews gesprochen) - dem Benutzer ist aber durchaus klar, dass hier keine normale Gesprächssituation vorliegt. Im Zweifel wird er sich deshalb sogar ganz anders als sonst verhalten und damit eine Vorlage für eine fehlerhafte Analyse liefern.

\subsection{Vorurteile und Verzerrungen}

In der ethischen und rechtlichen Diskussion von KI-Systemen ist oft von Vorurteilen oder Verzerrungen die Rede, von einem Bias. Meist handelt es sich um etwas, was unbewusst von Entwicklern ins System gegossen oder durch Big Data bzw. Machine Learning dort hinein gekommen ist. Der Bias kann z. B. das Geschlecht oder die Ethnie betreffen. Es werden falsche Annahmen getroffen und falsche Schlussfolgerungen gezogen, und zuweilen sind Unzulänglichkeiten bei der Analyse vorhanden. So sind Gesichts- und damit verbundene Emotionserkennungssysteme vor allem bei weißen Personen mehr oder weniger verlässlich, was zum einen an den Trainingsdaten liegt, zum anderen daran, dass weiße Gesichter von den verwendeten 
Technologien besser erkundet werden können als z.B. schwarze (Fernández und Fernández 2019).

Amazon entwickelte einen Chatbot, der ein Ranking für eingehende Bewerbungen generieren sollte, „mit dem Ziel, den Personalauswahlprozess zu optimieren“ (Mülder 2021). „Ein Jahr nach Inbetriebnahme stellte sich heraus, dass der Bot diskriminierend funktionierte und weibliche Bewerberinnen [!] beziehungsweise Bewerbungen mit dem Wort ,Women'schlechter einstufte als männliche Bewerber. Der Grund hierfür lag in den Datensätzen, mit denen trainiert wurde [...]“ (Mülder 2021) - ein Problem, das bereits oben angesprochen wurde. Basis waren Bewerbungen der vorangegangenen zehn Jahre, die mehrheitlich von männlichen Kandidaten stammten.

Im Prinzip können Vorurteile und Verzerrungen allerdings ebenso zurückgedrängt werden. Je nach Konzeption und Evolution der Systeme mögen sie objektiver sein als menschliche Recruiter und Interviewpartner. Sie können von Sympathie und Antipathie abstrahieren und weitere persönliche Gesichtspunkte außer Acht lassen. In dieser Richtung wollen Ethics by Design und Responsible AI wirken (Bendel 2021). Man vermeidet auf der Entwicklungsseite von Anfang an einen Bias im System oder erkennt einen solchen und beseitigt ihn dann oder gleicht ihn aus. Dabei helfen ethische Leitlinien, flankiert von Ethikkommissionen und -räten. Aus rechtlicher Sicht manifestieren sich Diskriminierungsaspekte, sodass das Antidiskriminierungsrecht greift (Imping 2021).

\subsection{Fehleinschätzungen, -informationen und -schlüsse}

Manche KI-Systeme, unter welchen Bedingungen und mit welchen Methoden auch immer, nehmen falsche Kategorisierungen vor, machen - wie bereits angedeutet falsche Aussagen und Vorhersagen bzw. liefern Fehlinformationen und Fehlschlüs$s e$. Diese können im vorliegenden Kontext zu Nachteilen für den Bewerber oder die Bewerberin führen, aber auch zu Vorteilen, was ethische, soziale und rechtliche Herausforderungen aufwirft. Beide Möglichkeiten sind problematisch, da eine Ungerechtigkeit zwischen Bewerbern erzeugt wird. Sie fördern zudem das Misstrauen gegenüber Systemen und Unternehmen.

Sind die Tools danach ausgelegt, eine Vorauswahl zu treffen, können Fehlschlüsse kaum korrigiert werden, wenn man erst gar nicht eingeladen und angehört wird. In diesem Sinne ist die Gefahr groß, dass sich ein fehlerhaftes System erhält und dem Unternehmen letztlich die geeigneten Kandidaten fehlen und es im „war of talents“ verliert. Es tauchen erneut Nachteile für Bewerber auf, wenn diese abgewiesen, und Vorteile, wenn sie durchgeschleust werden, was wiederum in Nachteile für das Unternehmen und seine Mitarbeiter münden kann.

\subsection{Profilbildung}

E-Assessments erlauben eine schnelle Bewertung des Benutzers, LinkedIn Learning und vergleichbare Plattformen und Kurse zudem eine längerfristige Bewertung und Einordnung. Ein Anbieter wie Microsoft (zu dem LinkedIn gehört) kann ein Profil erstellen, das Erfolge und Misserfolge festhält und sogar Rückschlüsse auf die In- 
telligenz zulässt - insbesondere die ethische und die rechtliche Perspektive sind hier gefragt. Auch zum Lernverhalten und zur Motivation können Aussagen gemacht werden. Dabei mangelt es meist an Kontextinformationen, etwa in welcher privaten Situation sich die Person befindet und in welchem physischen und psychischen $\mathrm{Zu}$ stand die Lektionen absolviert wurden, sodass verfälschte Erkenntnisse resultieren. Versucht das System allerdings, solche Kontextinformationen zu gewinnen, besteht wieder ein Problem mit personenbezogenen Daten.

Besonders heikel ist die Profilbildung, wenn sie bereits in jungen Jahren startet. Microsoft und Co. drängen seit vielen Jahren mit umstrittenen Mitteln und Allianzen in den Schulbereich (Bendel 2012). Sie haben zu Kindern und Jugendlichen, die die Produkte nutzen (müssen), umfassende Erkenntnisse. Diese könnten im Prinzip die Karriere im Guten wie im Schlechten beeinflussen. Während jedoch die Lehrkraft i. d.R. um die Gesamtsituation weiß und z.B. eine darauf abgestimmte Empfehlung für eine weiterführende Schule oder eine erste Ausbildung geben kann, ist dies dem System kaum möglich.

\subsection{Ungleichgewicht}

Ein weiteres Problem, das in der Informationsethik diskutiert wird, ist das Ungleichgewicht zwischen Betreiber und Benutzer, das sich auf verschiedenen Ebenen ausdrückt (Bendel 2012). Dieser verfügt nicht über die Technik des Betreibers, kennt die Reichweite und Funktionsweise nicht im Detail - etwa mit Blick auf Textanalyse - und ist sich unsicher, wie er sich darauf einstellen kann und soll. Er weiß nicht, an wen die Daten weitergereicht und vom wem sie eingesehen und ausgewertet werden. In vielen Fällen sind lediglich oberflächliche Informationen vorhanden. Als Betroffener ist man meist unterinformiert und wehrlos. Man kann sich allenfalls pauschal vorbereiten und versuchen, die Sprache des Unternehmens zu sprechen (CIO News 2021).

Das Ungleichgewicht kommt besonders dann zum Tragen, wenn eine missbräuchliche Verwendung innerhalb der Mitarbeiterschaft stattfindet. Dazu laden insbesondere Bilder ein. Sie können auf Plattformen gepostet oder mit Deep-Fake-Apps manipuliert werden. Emotionserkennung sowie Geschlechts- und Alterserkennung laden zu weiterem Missbrauch ein. Manche Systeme versprechen auch die Identifizierung individueller Haltungen und kulturellen Verhaltens durch E-Assessments sowie der politischen Gesinnung, der sexuellen Ausrichtung oder der kriminellen Energie durch Gesichtserkennung, ganz im Sinne traditioneller Physiognomik (Bendel 2019) und moderner Pseudowissenschaft.

Noch in anderer Hinsicht werden Unterschiede sichtbar. Die Nutzung von KISystemen kann im Moment, wie ausgeführt, mit hohen Anfangskosten verbunden sein. Nur bestimmte Unternehmen und letztlich nur bestimmte Länder sind in der Lage, ihre Bewerbungsprozesse damit effizienter und zumindest auf längere Sicht - dies ist nicht zuletzt von der Unternehmensgröße abhängig - effektiver zu gestalten. Damit entstehen anderen Unternehmen und Ländern allenfalls Nachteile, womöglich aber auch Vorteile, wenn sich ein menschengesteuertes Recruiting als effektiver (wenngleich weniger effizient) erweist. 


\section{Empfehlungen für Unternehmen}

Im Folgenden werden aus den vorausgegangenen Kapiteln Empfehlungen für Unternehmen und Organisationen für den Einsatz von KI-Systemen im Recruiting abgeleitet. Auch hierbei kann es sich um keine vollständige Liste handeln.

- An Datenschutzgesetze halten: Unternehmen, die KI-Systeme einsetzen, sollten sich über deren Funktionen im Detail informieren und die entsprechenden Regelungen - insbesondere die DSGVO mit ihren Ausführungen zur Erhebung und Verarbeitung von personenbezogenen Daten und speziell zu Privacy by Design und Privacy by Default - beachten (Bendel 2018b). Wenn eine Entscheidung ,automatisch" getroffen wird, ist nach DSGVO die Zustimmung des Betroffenen vonnöten (CIO News 2021).

- KI-Systeme sichern: Auch KI-Systeme können gehackt und manipuliert werden, von Personen außerhalb und innerhalb des Unternehmens. Entsprechend sind sie und die damit verbundenen Datenbanken, Wissensbasen und Informationssysteme abzusichern. Zudem ist auf die Vertrauenswürdigkeit und den Sitz der Anbieter zu achten.

- Ethische Leitlinien und rechtliche Rahmenbedingungen beachten: Auf EU-Ebene wurden Leitlinien für den Einsatz von KI-Systemen ${ }^{8}$ sowie rechtliche Rahmenbedingungen entwickelt. Diese gilt es zu beachten und einzubeziehen, im Einzelfall freilich herunterzubrechen und anzupassen. Als hohes Risiko wird im „legal framework on AI“ eine „CV sorting software for recruitment procedures“ (European Commission 2021) angesehen.

- Eigene Ethikkommission einrichten: Es können eigene Ethikkommissionen und passende Leitlinien etabliert werden. Ferner sind von mehreren Firmen genutzte Ethikkommissionen (etwa innerhalb von Branchen) denkbar. Es sollte kein reines Compliance-Management getätigt, sondern wissenschaftliche Ethik berücksichtigt werden, etwa um die diskursive Methode mit verschiedenen Interessengruppen anzuwenden und so einen Konsens zu erzielen (Kuhlen 2004).

- Beschäftigte einbeziehen: KI im Recruiting sollte nicht nur eine Aufgabe der HRAbteilung sein. Oliver Suchy, Mitglied des DGB sowie des Ethikbeirats für HR Tech, formuliert es so: „Wir müssen ganz am Anfang, im Ursprung der Entwicklung, Beschäftigte und Interessenvertretung beteiligen und gemeinsam diesen Prozess der Entwicklung von Zielen für eine KI, die Umsetzung und auch die Überprüfung neu organisieren" (Basso 2021). Am Ende müssen die Eintretenden und die Anwesenden miteinander arbeiten und auskommen.

- Awareness schaffen: Für die Chancen und Risiken und Fragen der Verantwortung ist bei der Belegschaft zu jedem Zeitpunkt der Einführung und des Betriebs der Recruitingsysteme Awareness zu schaffen. Die Systeme müssen gekannt, die Folgen beurteilt, im Zweifelsfall (auch angeordnete) Maßnahmen (etwa solche,

\footnotetext{
${ }^{8}$ Der Katalog der Empfehlungen der sogenannten High-level Expert Group on Artificial Intelligence (https://digital-strategy.ec.europa.eu/en/policies/expert-group-ai) war durchaus umstritten, zudem deren Zusammensetzung - philosophische Ethiker waren (im Gegensatz zu Vertretern der Industrie) kaum vertreten.
} 
die gegen die Compliance oder die Menschenwürde verstoßen) abgelehnt werden können.

- Schulungen anbieten: Mit Blick auf die Awareness, den technischen Hintergrund und die professionelle Handhabung der Recruitingsysteme sind den Beschäftigen regelmäßig Schulungen anzubieten, wobei ethische, soziale und rechtliche Aspekte relevant sind. Die Schulungen können eingekauft oder - dies ist vor allem sinnvoll, wenn Besonderheiten beim Einsatz auftreten - inhouse entwickelt werden.

- Transparenz herstellen: Für die Bewerber ist größtmögliche Transparenz herzustellen, wobei wiederum die Bestimmungen der DSGVO, aber ebenso unternehmenseigene (womöglich noch höhere) Standards herangezogen werden können. Sie sollten erfahren, dass (evtl. selbstlernende) Algorithmen verwendet werden, wie die Systeme funktionieren und was mit den persönlichen Daten geschieht. Sie sind rechtzeitig, umfassend und verständlich zu informieren.

- Alternativen anbieten: Die Bewerber sollten die Möglichkeit haben, ein traditionelles Verfahren zu durchlaufen oder bestimmte Verfahren im KI-gestützten Prozess wie Emotionserkennung ablehnen zu können, ohne dass ihnen daraus Nachteile resultieren. Dies kann wiederum im Kontext von Privacy by Design umgesetzt werden, und es sind einfache Auswahlmöglichkeiten wie Checkboxen und Menüs zu integrieren.

- Nachbereitung anschließen: Nach dem Bewerbungsgespräch bzw. dem Assessment sollte der Interessent die Möglichkeit haben, die umfeld- und personenbezogenen Daten im Zuge seiner informationellen Autonomie einzusehen - hier greift ebenfalls die DSGVO mit dem Recht auf Informationsfreiheit (als Informationszugangsfreiheit) - und ggf. zu löschen (wiederum im Sinne der DSGVO). Vor allem sollten auch Analyse und Interpretation bzw. maschinelle Entscheidungen erläutert werden.

\section{Zusammenfassung und Ausblick}

Der vorliegende Beitrag hat die technischen Basics und Potenziale des KI-gestützten Recruitings erklärt und wirtschaftliche Chancen und Risiken aufgezeigt. Ethische und soziale Herausforderungen wurden diskutiert und umsetzbare Handlungsempfehlungen für Unternehmen und Organisationen unterbreitet.

In einer Zukunftsvision können die technischen Möglichkeiten zur Motivation und automatisierten Verhaltensbeeinflussung (Nudging) dazu genutzt werden, nicht nur Kandidaten passiv auszuwählen, sondern deren Laufbahn aktiv im Sinne wirtschaftlicher Bedürfnisse zukunftsorientiert zu gestalten. Dies eröffnet ganz neue Perspektiven der Personalentwicklung für Unternehmen und erlaubt sogar eine aktive gemeinsame Gestaltung des Arbeitsmarkts für eine Branche oder ein ganzes Land.

Allerdings werden sich hier weitere rechtliche und ethische Herausforderungen ergeben. Es ist gefährlich, Arbeitskräfte nur als Humankapital zu betrachten, das man an sich ziehen und verwalten muss - durch Systeme, die viele der Betroffenen nicht verstehen können und nicht mögen werden. Es ist wichtig, die Motivation und 
das Vertrauen in jeder Phase des Anbahnungs- und Arbeitsverhältnisses aufrechtzuerhalten.

Funding Open access funding provided by FHNW University of Applied Sciences and Arts Northwestern Switzerland

Open Access Dieser Artikel wird unter der Creative Commons Namensnennung 4.0 International Lizenz veröffentlicht, welche die Nutzung, Vervielfältigung, Bearbeitung, Verbreitung und Wiedergabe in jeglichem Medium und Format erlaubt, sofern Sie den/die ursprünglichen Autor(en) und die Quelle ordnungsgemäß nennen, einen Link zur Creative Commons Lizenz beifügen und angeben, ob Änderungen vorgenommen wurden.

Die in diesem Artikel enthaltenen Bilder und sonstiges Drittmaterial unterliegen ebenfalls der genannten Creative Commons Lizenz, sofern sich aus der Abbildungslegende nichts anderes ergibt. Sofern das betreffende Material nicht unter der genannten Creative Commons Lizenz steht und die betreffende Handlung nicht nach gesetzlichen Vorschriften erlaubt ist, ist für die oben aufgeführten Weiterverwendungen des Materials die Einwilligung des jeweiligen Rechteinhabers einzuholen.

Weitere Details zur Lizenz entnehmen Sie bitte der Lizenzinformation auf http://creativecommons.org/ licenses/by/4.0/deed.de.

\section{Literatur}

Adamopoulou E, Moussiades L (2020) An overview of chatbot technology. In: Maglogiannis I, Iliadis L, Pimenidis E (Hrsg) Proceedings of AIAI 2020 Neos Marmaras, Greece, June 5-7. Artificial intelligence applications and innovations part II, S 373-383

Basso D (2021) KI in der Personalgewinnung. Personalmagazin 2021(7):82-83

Bendel O (2012) Die Rache der Nerds. UVK, Konstanz

Bendel O (2018a) The uncanny return of physiognomy. In: The 2018 AAAI spring symposium series. AAAI Press, Palo Alto, S 10-17

Bendel O (2018b) Datenschutz-Grundverordnung. In: Gabler Wirtschaftslexikon. Springer Gabler, Wiesbaden. https://wirtschaftslexikon.gabler.de/definition/datenschutz-grundverordnung-99476. Zugegriffen: 30. Dez. 2021

Bendel O (2019) Von Cor@ bis Mitsuku: Chatbots in der Kundenkommunikation und im Unterhaltungsbereich. In: Kollmann T (Hrsg) Handbuch Digitale Wirtschaft. Springer, Wiesbaden, S 1-17

Bendel O (2021) 300 Keywords Soziale Robotik: Soziale Roboter aus technischer, wirtschaftlicher und ethischer Perspektive. Springer Gabler, Wiesbaden

Black S, van Esch P (2020) AI-enabled recruiting: What is it and how should a manager use it? Bus Horiz 63(2):215-226

Campbell C, Sands S, Ferraro C, Tsao J, Mavrommatis A (2020) From data to action: how marketers can leverage AI. Bus Horiz 63(2):227-243

CIO News (2021) Den Lebenslauf für Algorithmen optimieren. CIO - IT-Strategie für Manager, September 2021

Deloitte (2018) The rise of the social enterprise. https://www2.deloitte.com/content/dam/insights/us/ articles/HCTrends2018/2018-HCtrends_Rise-of-the-social-enterprise.pdf. Zugegriffen: 30. Dez. 2021

European Commission (Hrsg) (2021) Excellence and trust in artificial intelligence. https://ec.europa.eu/ info/strategy/priorities-2019-2024/europe-fit-digital-age/excellence-trust-artificial-intelligence_en\# building-trust-through-the-first-ever-legal-framework-on-ai. Zugegriffen: 30. Dez. 2021

Fernández C, Fernández A (2019) Ethical and legal implications of AI recruiting software. ERCIM News 116:22-23 (Special theme: Transparency in Algorithmic Decision Making)

Guinan P, Parise S, Rollag K (2014) Jumpstarting the use of social technologies in your organization. Bus Horiz 57(3):337-347

Hickman L, Bosch N, Ng V, Saef R, Tay L, Woo S (2021) Automated video interview personality assessments: reliability, validity, and generalizability investigations. J Appl Psychol. https://doi.org/10. 1037/ap10000695 
Imping A (2021) Digitalisierung im Personalbereich: Rechtliche Rahmenbedingungen und Gestaltungsoptionen bei Betriebsvereinbarungen. Betrieb 32:1808-1818

Kosinski M, Wang Y (2017) Deep neural networks are more accurate than humans at detecting sexual orientation from facial images. https://psyarxiv.com/hv28a/. Zugegriffen: 30. Dez. 2021 (Journal of Personality and Social Psychology)

Kuhlen R (2004) Informationsethik: Umgang mit Wissen und Informationen in elektronischen Räumen. UVK/UTB, Konstanz

McIlvaine AR (2018) Data in the driver's seat. Human resource executive. https://hrexecutive.com/talentacquisitions-leaders-use-ai-to-improve-hiring/. Zugegriffen: 30. Dez. 2021

Mülder W (2021) Sind Chatbots die besseren Recruiter? Personalmagazin 2021(6):66-69

Norman W (1963) Toward an adequate taxonomy of personality attributes: replicated factor structure in peer nomination personality rating. J Abnorm Soc Psychol 66(6):574-583

Paschen U, Pitt C, Kietzmann J (2020) Artificial intelligence: building blocks and an innovation typology. Bus Horiz 63(2):215-226

Poole D, Mackworth A (2010) Artificial intelligence: foundations of computational agents. Cambridge University Press, Cambridge

Sharma A (2018) How AI reinvented hiring practice at L'Oréal. People Matters. https://www.peoplematters. in/article/techhr-2018/how-the-worlds-largest-cosmetic-company-transformed-its-hiring-practicewith-ai-19006. Zugegriffen: 30. Dez. 2021

Talent Board (2017) Talent board North American candidate experience research report. https://www. thetalentboard.org/press-releases/talent-board-2017-north-american-candidate-experience-researchreport-now-available. Zugegriffen: 30. Dez. 2021

Verhoeven T (Hrsg) (2020) Digitalisierung im Recruiting: Wie sich Recruiting durch künstliche Intelligenz, Algorithmen und Bots verändert. Springer, Wiesbaden, S 25-39 